\title{
Plumbagin, a natural naphthoquinone, inhibits the growth of esophageal squamous cell carcinoma cells through inactivation of STAT3
}

\author{
YAN CAO*, XIANG YIN*, YIPING JIA, BINGYAN LIU, SHAOQIU WU and MINGYI SHANG \\ Department of Interventional Radiology, Tongren Hospital, Shanghai Jiao Tong University \\ School of Medicine, Shanghai 200336, P.R. China
}

Received July 21, 2017; Accepted May 11, 2018

DOI: $10.3892 /$ ijmm.2018.3722

\begin{abstract}
Although plumbagin, a natural naphthoquinone, has exhibited antiproliferative activity in numerous types of cancer, its anticancer potential in esophageal squamous cell carcinoma (ESCC) remains unclear. In the present study, the effect of plumbagin on the growth of ESCC cells was investigated in vitro and in vivo. ESCC cells were treated with plumbagin and tested for cell cycle distribution and apoptosis. The involvement of STAT3 signaling in the effect of plumbagin was examined. The results demonstrated that plumbagin treatment suppressed ESCC cell viability and proliferation, yet normal esophageal epithelial cell viability was not affected. Plumbagin treatment increased the proportion of cells in the $\mathrm{G}_{0} / \mathrm{G}_{1}$ phase of the cell cycle and decreased the proportion of cells in the S phase. Furthermore, plumbagin-treated ESCC cells displayed a significantly greater \% of apoptotic cells. Western blot analysis confirmed that plumbagin upregulated tumor protein p53 and cyclin-dependent kinase inhibitor 1A (also known as p21), while it downregulated cyclin D1, cyclin-dependent kinase 4 , and induced myeloid leukemia cell differentiation protein Mcl-1. Mechanistically, plumbagin inhibited STAT3 activation, and overexpression of constitutively active STAT3 reversed the plumbagin-mediated growth suppression in ESCC cells. In vivo studies demonstrated that plumbagin delayed the growth of ESCC xenograft tumors and reduced STAT3 phosphorylation. Overall, plumbagin was demonstrated to target STAT3 signaling and to inhibit the
\end{abstract}

Correspondence to: Dr Shaoqiu Wu or Dr Mingyi Shang, Department of Interventional Radiology, Tongren Hospital, Shanghai Jiao Tong University School of Medicine, 1111 XianXia Road, Shanghai 200336, P.R. China

E-mail: shaoqiuwu@126.com

E-mail: ssFFR635987@163.com

${ }^{*}$ Contributed equally

Key words: esophageal cancer, naphthoquinone, signal transducer and activator of transcription 3, tumorigenesis growth of ESCC cells both in vitro and in vivo, suggesting that it may represent a potential anticancer agent for ESCC.

\section{Introduction}

Esophageal carcinoma, and in particular the histological subtype esophageal squamous cell carcinoma (ESCC), is frequently detected in developing countries including China $(1,2)$. At present, the main treatment options available for ESCC include radical esophagectomy, radiotherapy and chemotherapy. Despite advances in therapeutic approaches, the 5-year survival rate of ESCC remains very low $(3,4)$. Therefore, it is of importance to develop novel therapeutic modalities for ESCC.

Plumbagin (Fig. 1A) is a natural naphthoquinone that is widely distributed in the family of Plumbaginaceae. Plumbagin exhibits multiple biological properties, including antibacterial (5), antimalarial (6), anti-inflammatory (7), and antitumor (8) activities. It has been documented that plumbagin inhibits the invasion of breast cancer cells by inactivating signal transducer and activator of transcription 3 (STAT3) signaling (8). Likewise, plumbagin has been demonstrated to target the STAT3 pathway to block the growth of pancreatic cancer cells (9). STAT3 is a key transcription regulator that is aberrantly activated in ESCC $(10,11)$. It has been reported that knockdown of STAT3 reduces the proliferation of ESCC cells (11), indicating the requirement for STAT3 activation in the progression of ESCC. Given the inhibitory activity of plumbagin on STAT3 signaling, the present study hypothesized that plumbagin may exert anticancer effects against ESCC.

To test this hypothesis, in the present study ESCC cells were treated with different concentrations of plumbagin and the effects of plumbagin on cell proliferation, cell cycle progression and apoptosis were examined in vitro. In addition, the in vivo effect of plumbagin on the growth of ESCC xenograft tumors was investigated. Furthermore, the potential involvement of STAT3 signaling in the activity of plumbagin in ESCC cells was examined.

\section{Materials and methods}

Cell culture. Two human ESCC cell lines (ECA-109 and TE-1) were purchased from the Cell Bank of Type Culture 
Collection of the Chinese Academy of Sciences (Shanghai, China) and cultured in Dulbecco's modified Eagle's medium (DMEM; Invitrogen; Thermo Fisher Scientific, Inc., Waltham, MA, USA) supplemented with $10 \%$ fetal bovine serum (FBS; Sigma-Aldrich; Merck KGaA, Darmstadt, Germany) at $37^{\circ} \mathrm{C}$ in a $5 \% \mathrm{CO}_{2}$ atmosphere. Normal human esophageal epithelial cells were obtained from ScienCell Research Laboratories (San Diego, CA, USA) and maintained in Epithelial Cell Medium-2 (ScienCell Research Laboratories) containing $10 \%$ FBS.

Plumbagin treatment. Plumbagin ( $>95 \%$ in purity) was purchased from Sigma-Aldrich (Merck KGaA) and dissolved in dimethyl sulfoxide (DMSO; Sigma-Aldrich; Merck KGaA) to yield a stock solution of $20 \mathrm{mM}$. ECA-109 and TE-1 cells were exposed to different concentrations of plumbagin $(1-16 \mu \mathrm{M})(12)$ for $48 \mathrm{~h}$ and examined for viability, proliferation, cell cycle distribution and apoptosis. If not stated otherwise, $8 \mu \mathrm{M}$ of plumbagin was used in in vitro experiments.

Cell viability assay. Cell viability was determined using the 3-(4,5-dimethylthiazol-2-yl)-2,5-diphenyltetrazolium bromide (MTT) assay. In brief, following plumbagin treatment, cells were incubated with $0.5 \mathrm{mg} / \mathrm{ml}$ MTT (Sigma-Aldrich; Merck $\mathrm{KGaA}$ ) at $37^{\circ} \mathrm{C}$ for $4 \mathrm{~h}$, followed by the addition of DMSO. The absorbance of the MTT formazan reduction product was measured at $570 \mathrm{~nm}$.

Cell proliferation assay. Cell proliferation was measured using 5-bromo-2'-deoxyuridine (BrdU) incorporation assay (13). In brief, cells were labeled with BrdU (Roche Applied Science, Penzberg, Germany; $10 \mu \mathrm{M}$ ) during the last $4 \mathrm{~h}$ of plumbagin treatment. For quantification of BrdU incorporation, cells were incubated with anti-BrdU conjugated to peroxidase (Roche Applied Science), followed by addition of 3,3',5,5'-tetramethylbenzidine substrate solution (Roche Applied Science). The absorbance was measured at $450 \mathrm{~nm}$ using a microplate reader.

Cell cycle distribution and apoptosis analysis by flow cytometry. For analysis of cell cycle distribution, cells were fixed in ice-cold $70 \%$ ethanol and resuspended in staining solution containing DNase-free RNase A $(0.2 \mathrm{mg} / \mathrm{ml})$ and propidium iodide (PI; $20 \mu \mathrm{g} / \mathrm{ml}$ ), both from Sigma-Aldrich (Merck KGaA). Following incubation for $15 \mathrm{~min}$ in the dark, cellular DNA content was analyzed by flow cytometry. For measurement of apoptosis, cells were fixed and stained with fluorescein isothiocyanate (FITC)-conjugated Annexin V and PI using the Annexin V-FITC Apoptosis Detection kit (R\&D systems, Minneapolis, MN, USA). Stained cells were subjected to flow cytometric analysis using FlowJo software (version 10.0.4; Tree Star, Inc., Ashland, OR, USA).

Western blot analysis. Cells were lysed in ice-cold radioimmunoprecipitation assay (RIPA) buffer (Sigma-Aldrich; Merck KGaA) containing a mixture of protease inhibitors (Pierce; Thermo Fisher Scientific, Inc.). Protein concentrations were measured using the BCA assay kit (Pierce; Thermo Fisher Scientific, Inc.). Equal amounts of protein $(40 \mu \mathrm{g})$ were separated by SDS-polyacrylamide gel electrophoresis and transferred onto nitrocellulose membranes. Following blocking with 5\% fat-free milk, membranes were incubated with primary antibodies overnight at $4^{\circ} \mathrm{C}$ prior to incubation with horseradish peroxidase (HRP)-conjugated goat anti-mouse IgG (1:2,000; cat. no. sc-2005; Santa Cruz Biotechnology, Inc., Dallas, TX, USA) or HRP-conjugated goat anti-rabbit IgG (1:2,000; cat. no. sc-2030; Santa Cruz Biotechnology, Inc.) for $1 \mathrm{~h}$. The primary antibodies recognizing tumor protein p53 (cat. no. ab131442; 1:500), cyclin-dependent kinase inhibitor $1 \mathrm{~A}$ (also known as p21; cat. no. ab109520; 1:500), cyclin D1 (cat. no. ab134175; 1:500), cyclin-dependent kinase (CDK) 4 (cat. no. ab108357; 1:300), induced myeloid leukemia cell differentiation protein Mcl-1 (Mcl-1; cat. no. ab32087; 1:500), and $\beta$-actin (cat. no. ab8227; 1:2,000) were purchased from Abcam (Cambridge, UK). The antibodies against phospho-STAT3 (Tyr705; cat. no. 9131; 1:300), STAT3 (cat. no. 9132; 1:500), cleaved caspase-3 (cat. no. 9661; 1:300), and cleaved poly(ADP-ribose) polymerase (PARP; cat. no. 5625; 1:300) were from Cell Signaling Technology, Inc. (Danvers, MA, USA). Protein signals were visualized using enhanced chemiluminescence reagent (Amersham; GE Healthcare, Chicago, IL, USA) and quantified using Quantity One software (Bio-Rad Laboratories, Inc., Hercules, CA, USA).

Plasmids. A STAT3-responsive firefly luciferase plasmid (pSTAT3-Luc) was purchased from Panomics (Redwood City, CA, USA). A Renilla luciferase-expressing plasmid (pRL-TK) was purchased from Promega Corporation (Madison, WI, USA). A construct expressing constitutively active STAT3 mutant (pSTAT3-C) and empty vector were obtained from Addgene (Cambridge, MA, USA).

Cell transfection and luciferase reporter assay. For overexpression of constitutively active STAT3, ESCC cells were seeded $12 \mathrm{~h}$ prior to transfection. At $80 \%$ confluence, they were transfected with pSTAT3-C or empty vector using Lipofectamine 2000 following the manufacturer's instructions (Invitrogen; Thermo Fisher Scientific, Inc.). At $24 \mathrm{~h}$ after transfection, cells were treated with plumbagin and subjected to proliferation and apoptosis assays. For the luciferase reporter assay, ESCC cells were co-transfected with pSTAT3-Luc $(0.5 \mu \mathrm{g})$ and pRL-TK $(20 \mathrm{ng}) 24 \mathrm{~h}$ prior to plumbagin treatment. Following incubation for an additional $48 \mathrm{~h}$, cells were lysed and luciferase activities were measured using a luciferase assay kit (Promega Corporation). The firefly luciferase activity was normalized to that of Renilla luciferase.

Animal experiments. For xenograft studies, a total of 10 male nude mice (aged 4-6 weeks) were used, which were purchased from the Experimental Animal Center of Zhengzhou University (Zhengzhou, China). Animals were housed in a laminar air flow hood under a $12 / 12 \mathrm{~h}$ light/dark cycle at $25^{\circ} \mathrm{C}$ and $50 \%$ humidity with free access to food and water. ECA-109 and TE- 1 cells ( $2 \times 10^{6}$ cells/mouse) were subcutaneously injected into the flanks of mice. When xenograft tumors reached $100 \mathrm{~mm}^{3}$, the tumor-bearing mice ( $\mathrm{n}=5$ for each group) were intraperitoneally administered with plumbagin or vehicle (0.05\% DMSO) at a dose of $2 \mathrm{mg} / \mathrm{kg} / \mathrm{day} 3$ times 
A<smiles>CC1=CC(=O)c2c(O)cccc2C1=O</smiles>

Plumbagin

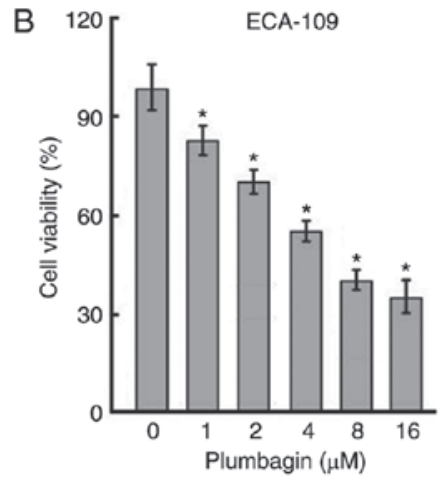

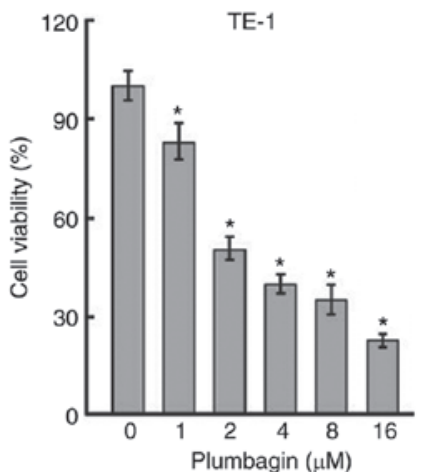

Normal cells
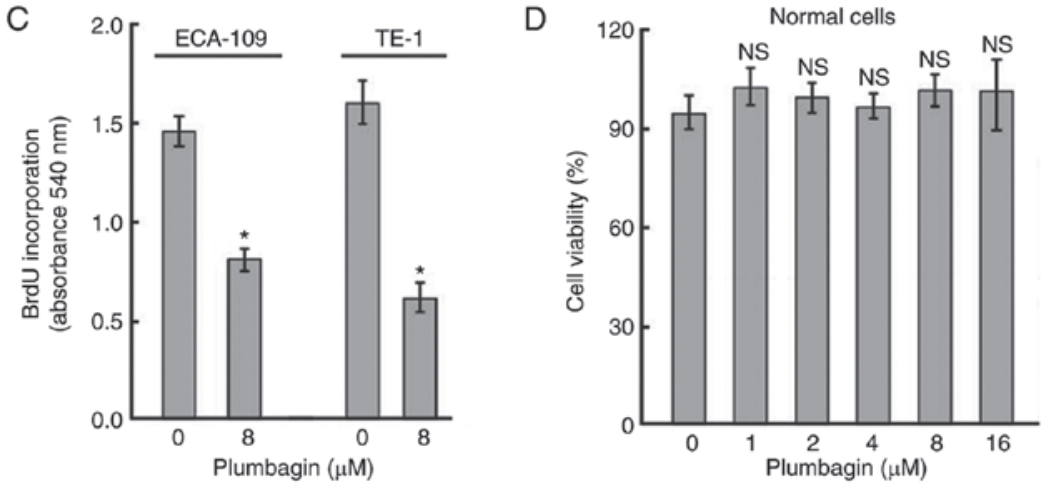

Figure 1. Plumbagin inhibits the viability and proliferation of esophageal squamous cell carcinoma cells in vitro. (A) Chemical structure of plumbagin. (B) ECA-109 and TE-1 cells were exposed to different concentrations of plumbagin for $48 \mathrm{~h}$ and cell viability was determined by MTT assay. (C) ECA-109 and TE-1 cells were treated with $8 \mu \mathrm{M}$ of plumbagin or vehicle for $48 \mathrm{~h}$ and cell proliferation was assessed using BrdU incorporation assay. (D) Measurement of the viability of normal esophageal epithelial cells following treatment with plumbagin for $48 \mathrm{~h}$. $\mathrm{P}<0.05$ compared with vehicle-treated cells. NS, not significant.

per week for 4 weeks (14). Tumor growth was monitored by measuring tumor volume. All animals were sacrificed after 30 days of treatment. Tumors were removed and processed for immunohistochemical staining using anti-Ki-67 (cat. no. ab15580; 1:500; Abcam) and anti-phospho-STAT3 (1:100) antibodies (15). Over 1,000 cells were counted from 5 random microscopic fields, and the $\%$ of immunoreactive cells was calculated. All experiments involving animals were approved by the Experimental Animal Ethics Committee of Shanghai Jiao Tong University School of Medicine (Shanghai, China; Approval number: 2016-01-032).

Statistical analysis. Data are presented as the mean \pm standard deviation. Statistical differences were determined using the Student's $t$-test for comparison of two groups or one-way analysis of variance followed by Tukey's post-hoc test for multiple comparisons. $\mathrm{P}<0.05$ was considered to indicate a statistically significant difference.

\section{Results}

Plumbagin inhibits the viability and proliferation of ESCC cells in vitro. To assess the cytotoxicity of plumbagin, two ESCC cell lines were exposed to different concentrations of plumbagin for $48 \mathrm{~h}$. As determined by MTT assay (Fig. 1B), plumbagin treatment resulted in a concentration-dependent reduction in cell viability. The $\mathrm{IC}_{50}$ values for plumbagin in ECA-109 and TE-1 cells were 6.2 \pm 0.5 and $2.4 \pm 0.6 \mu \mathrm{M}$, respectively. BrdU incorporation assay was preformed to validate the antiproliferative activity of plumbagin. The results demonstrated that treatment with $8 \mu \mathrm{M}$ plumbagin for $48 \mathrm{~h}$ decreased the proliferation of ECA-109 and TE-1 cells by 45.5 and $62.6 \%$, respectively (Fig. 1C). However, plumbagin, up to the concentration of $16 \mu \mathrm{M}$, had no significant impact on the viability of normal esophageal epithelial cells following treatment for $48 \mathrm{~h}$ (Fig. 1D).

Plumbagin induces cell cycle arrest at the $G_{0} / G_{I}$ phase and triggers apoptosis in ESCC cells. Flow cytometric analysis was performed to determine the effect of plumbagin on cell cycle progression and apoptosis. As presented in Fig. 2A, treatment of ECA-109 cells with plumbagin for $48 \mathrm{~h}$ increased the proportion of cells in the $G_{0} / G_{1}$ phase of the cell cycle from 50 to $62 \%(\mathrm{P}=0.001)$ and decreased the proportion of cells in the $\mathrm{S}$ phase from 29 to $18 \%(\mathrm{P}=0.002)$ relative to control. In addition, plumbagin treatment resulted in a 4.7-fold $(\mathrm{P}=0.004)$ increase in the $\%$ of Annexin V-positive apoptotic cells, compared with vehicle-treated cells (Fig. 2B). Similar findings were observed in TE-1 cells following treatment with plumbagin (Fig. 2). Western blot analysis of several key genes involved in cell cycle progression and apoptosis confirmed that plumbagin treatment resulted in a marked increase in p53 and p21 and decrease in cyclin D1, CDK4 and Mcl-1 expression (Fig. 3A). In addition, cleavage of caspase-3 and PARP was markedly enhanced in plumbagin-treated ECA-109 and TE-1 cells compared with vehicle-treated cells (Fig. 3A).

Plumbagin inhibits the STAT3 signaling pathway. Western blot analysis demonstrated that plumbagin treatment led to a significant decrease in the levels of phosphorylated 

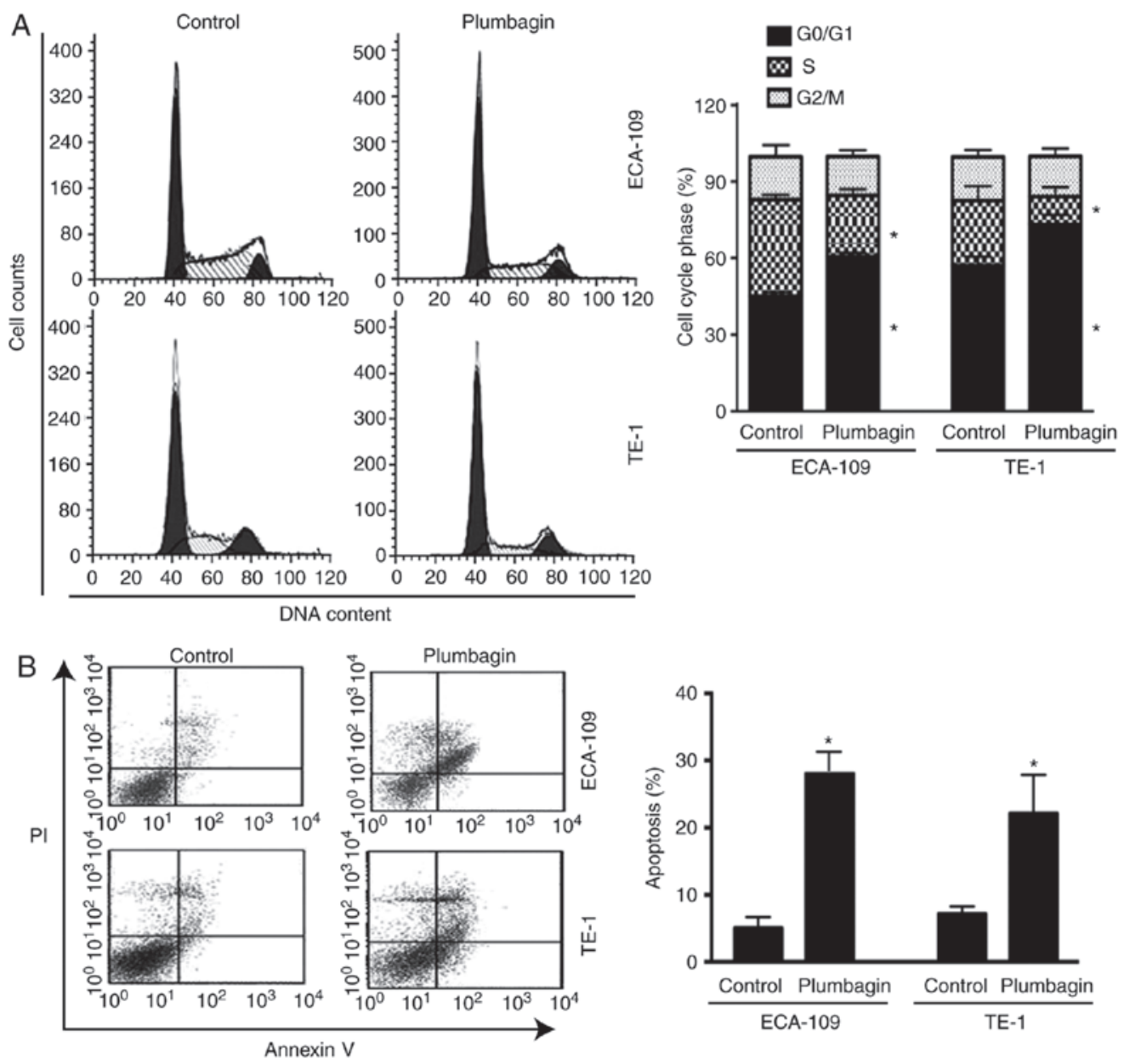

Figure 2. Plumbagin induces cell cycle arrest at the $\mathrm{G}_{0} / \mathrm{G}_{1}$ phase and triggers apoptosis in esophageal squamous cell carcinoma cells. ECA-109 and TE-1 cells were treated with $8 \mu \mathrm{M}$ of plumbagin or vehicle for $48 \mathrm{~h}$. (A) Cell cycle distribution was examined by flow cytometric analysis of DNA content after PI staining. (B) Apoptosis was examined by flow cytometric analysis following Annexin V/PI staining. "P<0.05 compared with vehicle-treated cells. PI, propidium iodide.

STAT3, but not total STAT3 protein (Fig. 3B). Consistently, plumbagin-treated ESCC cells displayed a 3- to 6-fold reduction of STAT3 reporter gene activity, compared with control cells (Fig. 3C). These data indicated that plumbagin had the capacity to interfere with STAT3 activation.

Overexpression of constitutively active STAT3 reverses plumbagin-mediated growth suppression. Next, the hypothesis that the plumbagin-mediated cytotoxicity in ESCC cells was directly associated with inactivation of STAT3 signaling was examined. To this end, rescue experiments were performed with a constitutively active STAT3 variant. The results demonstrated that ectopic expression of constitutively active STAT3 (Fig. 4A) significantly attenuated the plumbagin-induced growth suppression (Fig. 4B) and apoptosis (Fig. 4C) in ESCC cells.

Plumbagin suppresses the growth of ESCC xenografts in mice. To determine the in vivo anticancer effect of plumbagin, ECA-109 and TE-1 cells were injected into nude mice, allowed to form xenograft tumors, and treated with plumbagin or vehicle in vivo. After 30 days, plumbagin-treated ECA-109 and TE-1 xenograft tumors displayed 72 and 54\% reduction in tumor volume, respectively, relative to vehicle-treated tumors (Fig. 5A). Immunohistochemistry analysis of tumor specimens confirmed that plumbagin-treated tumors presented a significant reduction in the $\%$ of $\mathrm{Ki}-67$-positive cells $(\mathrm{P}=0.011$; Fig. 5B). Additionally, the levels of phosphorylated STAT3 were significantly lower in plumbagin-treated tumors compared with vehicle-treated tumors $(\mathrm{P}=0.001$; Fig. 5C).

\section{Discussion}

Plumbagin has exhibited anticancer properties in many types of cancer, including breast cancer (8), pancreatic cancer (9), prostate cancer (16), and gastric cancer (17). It has been reported that plumbagin can block prostate carcinogenesis triggered by loss of phosphatase and tensin homolog (PTEN) (16). Similarly, plumbagin treatment resulted in a significant inhibition of tumorigenesis and angiogenesis in ovarian cancer xenograft models (14). In the present study, plumbagin was also confirmed to exert growth suppressive effects against ESCC cells. Treatment with plumbagin led to a significant decline in cell viability and proliferation in ESCC cells. Plumbagin-mediated toxicity appeared to be selective to malignant cells, as the viability of a normal esophageal epithelial cell line was not significantly changed following plumbagin treatment. In agreement with the present findings, 

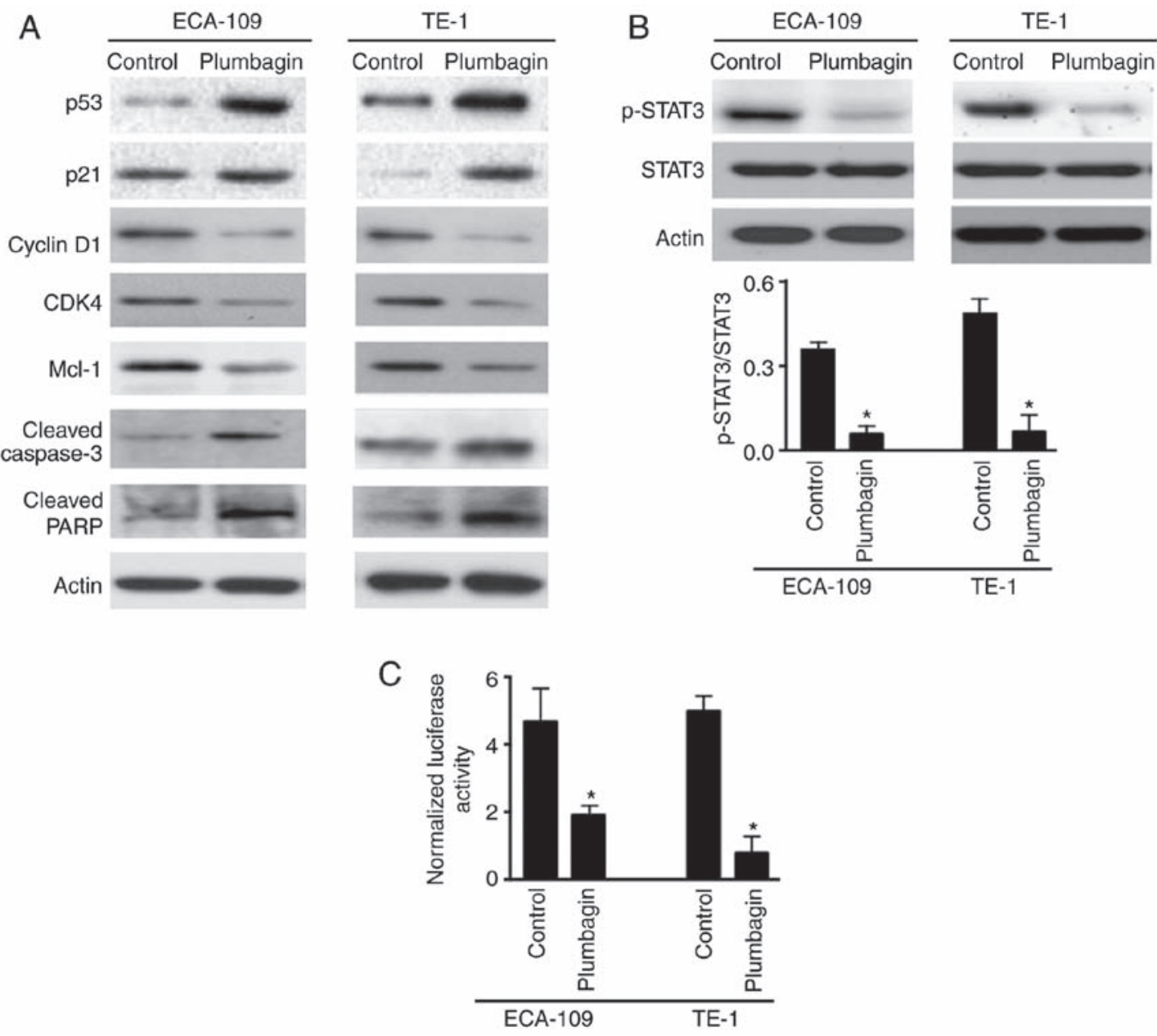

Figure 3. Plumbagin treatment affects the expression of proteins involved in cell cycle progression and apoptosis and inhibits the STAT3 signaling pathway. ESCC cells were treated with $8 \mu \mathrm{M}$ of plumbagin or vehicle (control) for $48 \mathrm{~h}$. (A) Western blot analysis of indicated proteins in ESCC cells. Representative blots are shown from three independent experiments. (B) Western blot analysis and quantification of STAT3 phosphorylation in treated ESCC cells. (C) STAT3-dependent luciferase reporter assay. ESCC cells were co-transfected with STAT3 luciferase reporter (0.5 $\mu \mathrm{g})$ and pRL-TK (20 ng) $24 \mathrm{~h}$ prior to plumbagin treatment. The firefly luciferase activity was normalized to that of Renilla luciferase. " $\mathrm{P}<0.05$ compared with vehicle-treated control cells. ESCC, esophageal squamous cell carcinoma; STAT3, signal transducer and activator of transcription 3; p53, tumor protein p53; p21, cyclin-dependent kinase inhibitor 1A; CDK4; cyclin-dependent kinase 4; Mcl-1; induced myeloid leukemia cell differentiation protein Mcl-1; Casp-3, caspase-3; PARP, poly(ADP-ribose) polymerase; p-, phosphorylated.
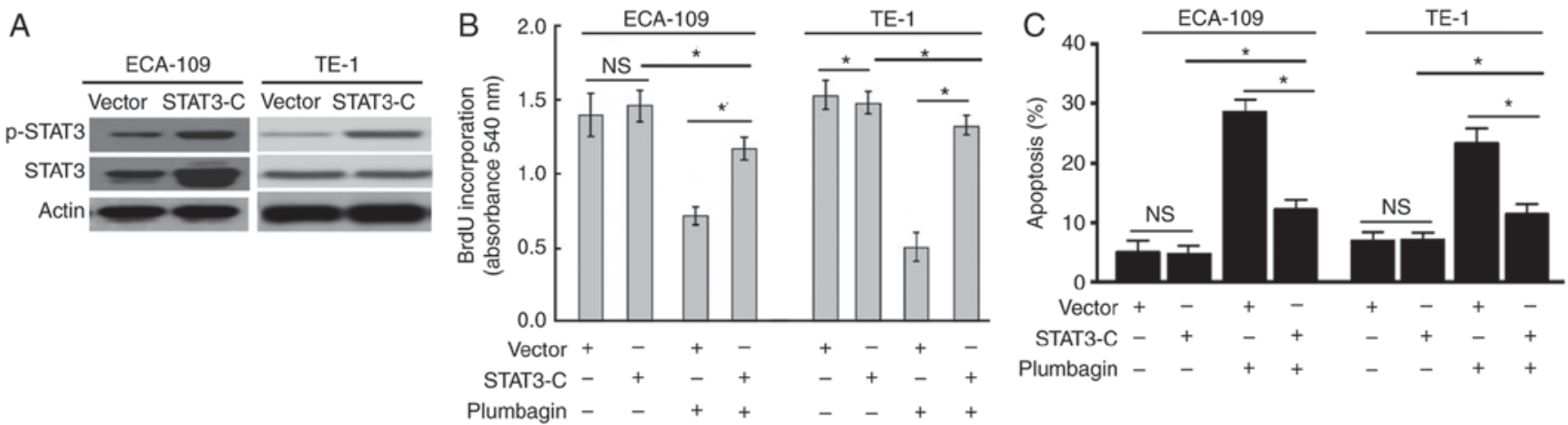

Figure 4. Overexpression of constitutively active STAT3 reverses the plumbagin-mediated growth suppression. (A) Western blot analysis of STAT3 phosphorylation in ESCC cells transfected with a plasmid expressing constitutively active STAT3 (STAT3-C) or empty vector. (B and C) ESCC cells were transfected with STAT3-C or empty vector prior to treatment with $8 \mu \mathrm{M}$ of plumbagin for $48 \mathrm{~h}$. BrdU incorporation assay was performed to determine cell proliferation, and flow cytometry analysis following Annexin V/propidium iodide staining was performed to determine apoptosis. " $\mathrm{P}<0.05$ with comparisons indicated by lines. STAT3, signal transducer and activator of transcription 3; ESCC, esophageal squamous cell carcinoma; p-, phosphorylated; NS, not significant.

a previous study has demonstrated that plumbagin was more effective at killing melanoma cells than non-malignant cells in vitro and that it displayed negligible toxicity to normal organs in vivo (18). 
A

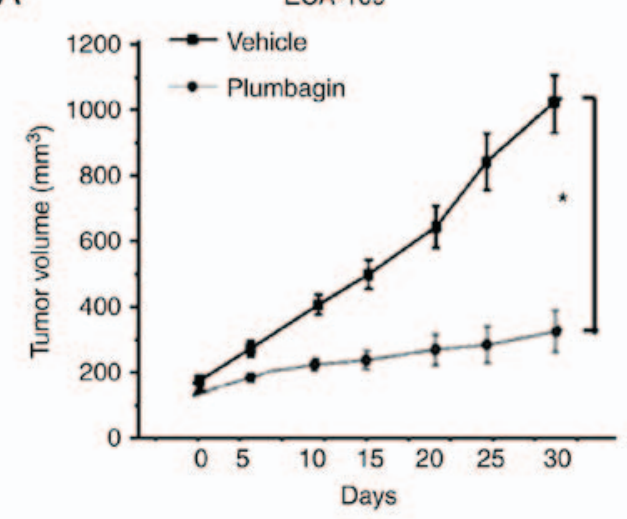

TE-1

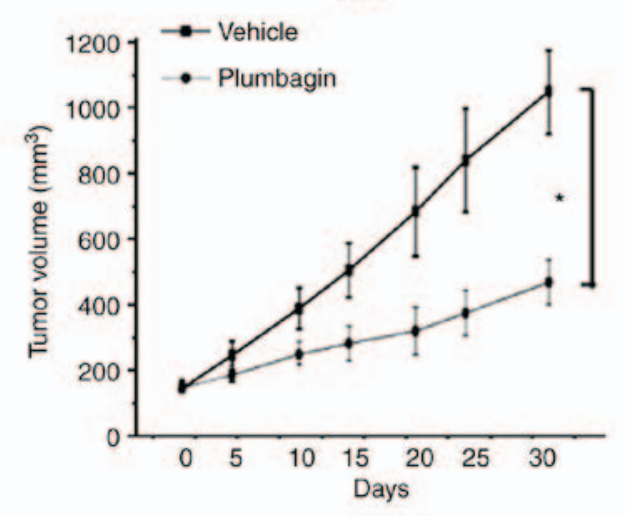

B
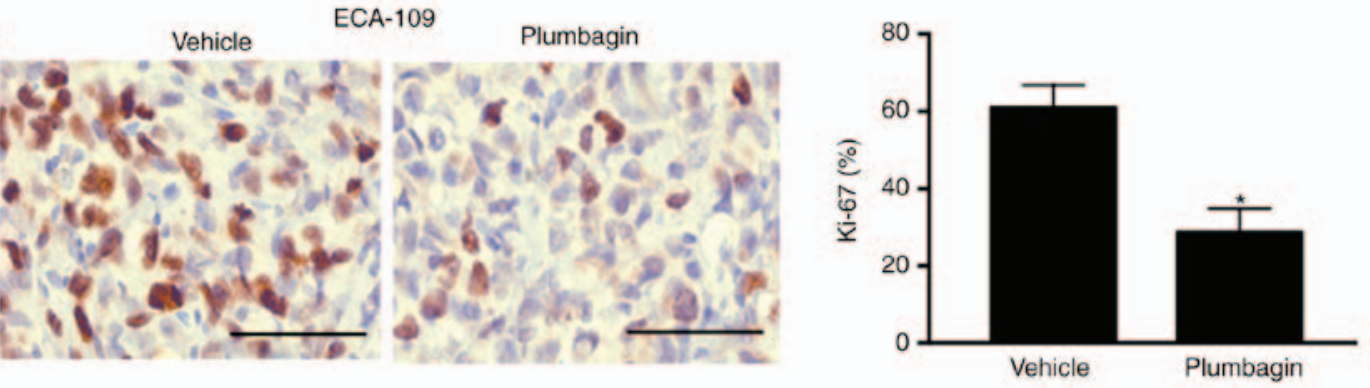

C
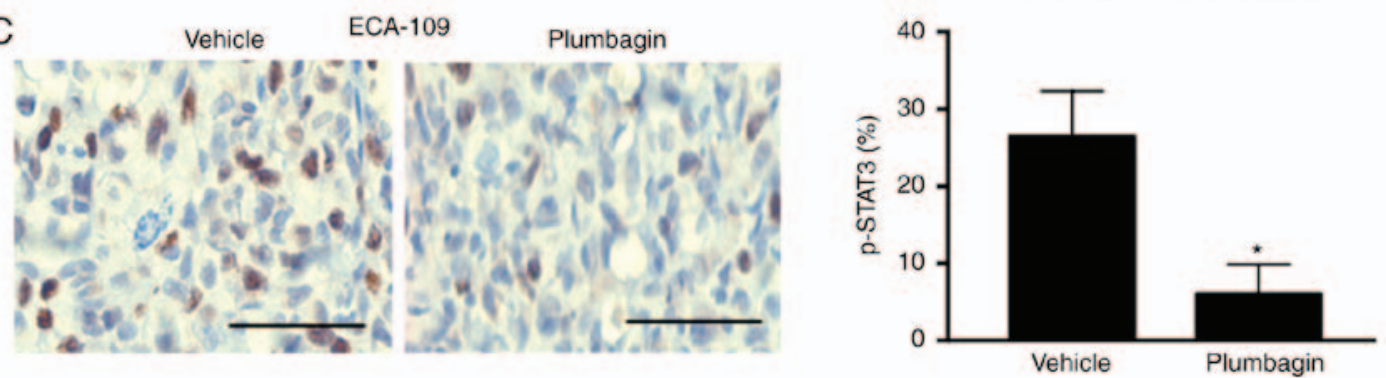

Figure 5. Plumbagin suppresses the growth of esophageal squamous cell carcinoma xenografts in mice. ECA-109 and TE-1 cells were injected into nude mice, allowed to form xenograft tumors, and then treated with plumbagin $(2 \mathrm{mg} / \mathrm{kg} /$ day) or vehicle for a total of 30 days. (A) Growth curves of the xenograft tumors in each treatment group. (B) Immunohistochemistry analysis of tumor sections for Ki-67 and (C) p-STAT3 levels. The \% of Ki-67 or p-STAT3-positive cells over total was determined. Scale bar, $60 \mu \mathrm{m}$. "P<0.05 compared with the vehicle-treated group. p-, phosphorylated; STAT3, signal transducer and activator of transcription 3.

To gain more insight into the growth suppression induced by plumbagin, the effects of plumbagin on cell cycle distribution and apoptosis were analyzed. Plumbagin-treated ESCC cells displayed an accumulation of $G_{0} / G_{1}$-phase cells and significant apoptotic death, compared with vehicle-treated cells. At the molecular level, multiple genes, including p53, p21, cyclin D1, CDK4 and Mcl-1 were deregulated in response to plumbagin treatment. The protein $\mathrm{p} 21$ is a well-defined CDK inhibitor that can bind to cyclin/CDK complexes and inhibit their catalytic activities (19). p53 acts as a tumor suppressor and has the ability to induce cell cycle arrest and apoptosis (20), while Mcl-1 serves an important role in protecting cells from apoptosis (21). Taken together, plumbagin treatment was demonstrated to modulate many key genes involved in the regulation of cell cycle progression and apoptosis, consequently leading to cell cycle arrest and apoptosis. Such effects were also previously reported in breast cancer (22) and colon cancer cells (23). It should be mentioned that the suppressive activity of plumbagin appears not to be $\mathrm{G}_{1}$-phase specific, as this compound can cause $\mathrm{G}_{2} / \mathrm{M}$-phase arrest in A549 lung cancer cells (24).
Targeting STAT3 signaling represents an important molecular mechanism for the anticancer activity of plumbagin in melanoma (18), gastric cancer (17), breast cancer (8), and prostate cancer (25). In line with these studies, the present data demonstrated that plumbagin treatment significantly suppressed STAT3 activation in ESCC cells. STAT3 has been reported to regulate many genes involved in cell proliferation and survival, including cyclin D1, Bcl-2, Mcl-1, and cellular inhibitor of apoptosis 2 (c-IAP2) $(26,27)$. Consistent with the inactivation of STAT3, the expression of cyclin D1 and Mcl-1 was downregulated in ESCC cells in response to plumbagin treatment. Rescue experiments provided further evidence for the involvement of STAT3 signaling in the anticancer activity of plumbagin in ESCC. In vivo studies demonstrated that plumbagin treatment significantly delayed the growth of established ESCC xenograft tumors and suppressed STAT3 phosphorylation. Collectively, plumbagin exerted its growth suppressive activity in ESCC by targeting STAT3 signaling, which may provide an explanation for selective inhibition of cancer 
cells. It was reported that plumbagin can inhibit A549 lung cancer cell growth by inactivating c-Jun N-terminal kinase signaling (24). Therefore, it is possible that multiple signaling pathways other than STAT3 are involved in the anticancer activity of plumbagin in ESCC.

In conclusion, the present data reinforce that plumbagin has growth suppressive activity against ESCC cells, which is, at least in part, mediated through inhibition of STAT3 activation. These findings suggest that plumbagin may be a promising anticancer agent for ESCC.

\section{Acknowledgements}

Not applicable.

\section{Funding}

This work was funded by the Research Program of Shanghai Health and Family Planning Commission of China (grant no. 20134036).

\section{Availability of data and materials}

The analyzed datasets generated during the study are available from the corresponding author on reasonable request.

\section{Authors' contributions}

YC and XY were responsible for study design, data collection and analysis. YJ, BL and SW performed in vivo studies and histological examination. MS performed in vitro studies and statistical analysis. All authors read and approved the final manuscript.

\section{Ethics approval and consent to participate}

All experiments involving animals were approved by the Experimental Animal Ethics Committee of Shanghai Jiao Tong University School of Medicine (Shanghai, China; Approval number, 2016-01-032).

\section{Patient consent for publication}

Not applicable.

\section{Competing interests}

The authors declare that they have no competing interests.

\section{References}

1. Siegel RL, Miller KD and Jemal A: Cancer statistics, 2015. CA Cancer J Clin 65: 5-29, 2015.

2. Chen W, Zheng R, Baade PD, Zhang S, Zeng H, Bray F, Jemal A, Yu XQ and He J: Cancer statistics in China, 2015. CA Cancer J Clin 66: 115-132, 2016.

3. Shridhar R, Hoffe SE, Almhanna K, Weber JM, Chuong MD, Karl RC and Meredith K: Lymph node harvest in esophageal cancer after neoadjuvant chemoradiotherapy. Ann Surg Oncol 20: 3038-3043, 2013.

4. Jackie Oh S, Han S, Lee W and Lockhart AC: Emerging immunotherapy for the treatment of esophageal cancer. Expert Opin Investig Drugs 25: 667-677, 2016.
5. Omosa LK, Midiwo JO, Mbaveng AT, Tankeo SB, Seukep JA, Voukeng IK, Dzotam JK, Isemeki J, Derese S, Omolle RA, et al: Antibacterial activities and structure-activity relationships of a panel of 48 compounds from Kenyan plants against multidrug resistant phenotypes. Springerplus 5: 901, 2016.

6. Sumsakul W, Chaijaroenkul W and Na-Bangchang K: In vitro inhibitory effects of plumbagin, the promising antimalarial candidate, on human cytochrome P450 enzymes. Asian Pac J Trop Med 8: 914-918, 2015.

7. Pile JE, Navalta JW, Davis CD and Sharma NC: Interventional effects of plumbagin on experimental ulcerative colitis in mice. J Nat Prod 76: 1001-1006, 2013.

8. Yan W, Tu B, Liu YY, Wang TY, Qiao H, Zhai ZJ, Li HW and Tang TT: Suppressive effects of plumbagin on invasion and migration of breast cancer cells via the inhibition of STAT3 signaling and down-regulation of inflammatory cytokine expressions. Bone Res 1: 362-370, 2013.

9. Hafeez BB, Jamal MS, Fischer JW, Mustafa A and Verma AK: Plumbagin, a plant derived natural agent inhibits the growth of pancreatic cancer cells in in vitro and in vivo via targeting EGFR, Stat 3 and NF- $\kappa$ B signaling pathways. Int J Cancer 131: 2175-2186, 2012.

10. Zhang Y, Du XL, Wang CJ, Lin DC, Ruan X, Feng YB, Huo YQ, Peng H, Cui JL, Zhang TT, et al: Reciprocal activation between PLK1 and Stat 3 contributes to survival and proliferation of esophageal cancer cells. Gastroenterology 142: 521-530, 2012.

11. Timme S, Ihde S, Fichter CD, Waehle V, Bogatyreva L, Atanasov K, Kohler I, Schöpflin A, Geddert H, Faller G, et al: STAT3 expression, activity and functional consequences of STAT3 inhibition in esophageal squamous cell carcinomas and Barrett's adenocarcinomas. Oncogene 33: 3256-3266, 2014.

12. Niu M, Cai W, Liu H, Chong Y, Hu W, Gao S, Shi Q, Zhou X, Liu X and Yu R: Plumbagin inhibits growth of gliomas in vivo via suppression of FOXM1 expression. J Pharmacol Sci 128: 131-136, 2015.

13. Radestock Y, Hoang-Vu C and Hombach-Klonisch S: Relaxin reduces xenograft tumour growth of human MDA-MB-231 breast cancer cells. Breast Cancer Res 10: R71, 2008.

14. Sinha S, Pal K, Elkhanany A, Dutta S, Cao Y, Mondal G, Iyer S, Somasundaram V, Couch FJ, Shridhar V, et al: Plumbagin inhibits tumorigenesis and angiogenesis of ovarian cancer cells in vivo. Int J Cancer 132: 1201-1212, 2013.

15. Liu C, Dai LH, Dou DQ, Ma LQ and Sun YX: A natural food sweetener with anti-pancreatic cancer properties. Oncogenesis 5: e217, 2016.

16. Hafeez BB, Fischer JW, Singh A, Zhong W, Mustafa A, Meske L, Sheikhani MO and Verma AK: Plumbagin inhibits prostate carcinogenesis in intact and castrated PTEN knockout mice via targeting $\mathrm{PKC} \varepsilon$, Stat 3 , and epithelial-to-mesenchymal transition markers. Cancer Prev Res (Phila) 8: 375-386, 2015.

17. Joo MK, Park JJ, Kim SH, Yoo HS, Lee BJ, Chun HJ, Lee SW and Bak YT: Antitumorigenic effect of plumbagin by induction of SH2-containing protein tyrosine phosphatase 1 in human gastric cancer cells. Int J Oncol 46: 2380-2388, 2015.

18. Gowda R, Sharma A and Robertson GP: Synergistic inhibitory effects of Celecoxib and Plumbagin on melanoma tumor growth. Cancer Lett 385: 243-250, 2017.

19. Coqueret O: New roles for p21 and p27 cell-cycle inhibitors: A function for each cell compartment? Trends Cell Biol 13: 65-70, 2003.

20. Vousden KH and Lu X: Live or let die: The cell's response to p53. Nat Rev Cancer 2: 594-604, 2002.

21. Morciano G, Pedriali G, Sbano L, Iannitti T, Giorgi C and Pinton P: Intersection of mitochondrial fission and fusion machinery with apoptotic pathways: Role of Mcl-1. Biol Cell 108: 279-293, 2016.

22. Zhang XQ, Yang CY, Rao XF and Xiong JP: Plumbagin shows anti-cancer activity in human breast cancer cells by the upregulation of p53 and p21 and suppression of G1 cell cycle regulators. Eur J Gynaecol Oncol 37: 30-35, 2016.

23. Eldhose B, Gunawan M, Rahman M, Latha MS and Notario V: Plumbagin reduces human colon cancer cell survival by inducing cell cycle arrest and mitochondria-mediated apoptosis. Int J Oncol 45: 1913-1920, 2014. 
24. Hsu YL, Cho CY, Kuo PL, Huang YT and Lin CC: Plumbagin (5-hydroxy-2-methyl-1,4-naphthoquinone) induces apoptosis and cell cycle arrest in A549 cells through p53 accumulation via c-Jun NH2-terminal kinase-mediated phosphorylation at serine 15 in vitro and in vivo. J Pharmacol Exp Ther 318: 484-494, 2006.

25. Hafeez BB, Zhong W, Fischer JW, Mustafa A, Shi X, Meske L, Hong H, Cai W, Havighurst T, Kim K and Verma AK: Plumbagin, a medicinal plant (Plumbago zeylanica)-derived 1,4-naphthoquinone, inhibits growth and metastasis of human prostate cancer PC-3M-luciferase cells in an orthotopic xenograft mouse model. Mol Oncol 7: 428-439, 2013.
26. Leslie K, Lang C, Devgan G, Azare J, Berishaj M, Gerald W, Kim YB, Paz K, Darnell JE, Albanese C, et al: Cyclin D1 is transcriptionally regulated by and required for transformation by activated signal transducer and activator of transcription 3 . Cancer Res 66: 2544-2552, 2006.

27. Bhattacharya S, Ray RM and Johnson LR: STAT3-mediated transcription of $\mathrm{Bcl}-2, \mathrm{Mcl}-1$ and $\mathrm{c}-\mathrm{IAP} 2$ prevents apoptosis in polyamine-depleted cells. Biochem J 392: 335-344, 2005. 